Published in final edited form as:

J Cell Biochem. 2014 May ; 115(5): 889-895. doi:10.1002/jcb.24730.

\title{
Down-Regulation of miR-96 by Bone Morphogenetic Protein Signaling is Critical for Vascular Smooth Muscle Cell Phenotype Modulation ${ }^{\prime}$
}

\author{
Sunghwan Kim ${ }^{1}$, Akiko Hata ${ }^{2}$, and Hara Kang ${ }^{3,}{ }^{*}$ \\ ${ }^{1}$ Laboratory of Dermatology-Immunology, College of Medicine, Catholic University of Korea, \\ Seoul, Republic of Korea \\ ${ }^{2}$ Cardiovascular Research Institute, University of California at San Francisco, San Francisco, CA \\ 94158, USA \\ ${ }^{3}$ Division of Life Sciences, College of Life Sciences and Bioengineering, Incheon National \\ University, Incheon 406-772, Republic of Korea
}

\begin{abstract}
The bone morphogenetic protein (BMP) signaling pathway is critical for the induction and maintenance of contractile phenotype in vascular smooth muscle cells (VSMCs). Inactivation of BMP signaling is common in abnormalities in vascular development and in vascular proliferative conditions, such as pulmonary artery hypertension. Herein, we identify microRNA-96 (miR-96) as a modulator of the VSMC phenotype in response to BMP4 signaling. We show that miR-96 is down-regulated by BMP4 treatment, which results in the derepression of a novel target, Tribbleslike protein 3 (Trb3). miR-96 targets a partially complementary sequence localized in the 3' UTR of Trb3. Trb3 is an essential positive regulator of the BMP signaling pathway and promotes contractile phenotype in VSMCs. In conclusion, our study demonstrates a novel mechanism of regulation of SMC-specific gene expression and induction of a VSMC contractile phenotype by the BMP4 signaling pathway via suppression of the miR-96-Trb3 axis.
\end{abstract}

\section{Keywords}

Bone morphogenetic protein; vascular smooth muscle cells; microRNA-96; Tribbles-like protein 3

\section{Introduction}

The transforming growth factor $\beta$ (TGF $\beta$ ) family of growth factors plays an essential role during vascular development and maintenance of homeostasis [1,2]. TGF $\beta$ and BMP have been shown to modulate the phenotypes of VSMCs. VSMCs retain the ability to switch

\footnotetext{
$\dagger$ This article has been accepted for publication and undergone full peer review but has not been through the copyediting, typesetting, pagination and proofreading process, which may lead to differences between this version and the Version of Record. Please cite this article as doi: [10.1002/jcb.24730]

*To whom correspondence should be addressed: Hara Kang, PhD., at Division of Life Sciences, College of Life Sciences and Bioengineering, Incheon National University, Incheon 406-772, Republic of Korea, Tel. +82-32-835-8238 Fax: +82-32-835-0763, harakang@incheon.ac.kr.
} 
between differentiated "contractile" and dedifferentiated "synthetic" phenotypes in response to environmental signals [3]. TGF $\beta$ and BMP inhibit VSMC proliferation and migration and increase contractile VSMC gene expression, which in turn promote the contractile phenotype and inhibit switching to the synthetic phenotype. Aberrant signaling pathways of TGF $\beta$ and BMP have been linked to vascular disorders, such as pulmonary artery hypertension (PAH) and hereditary hemorrhagic telangiectasia [4].

In VSMCs, TGF $\beta$ and BMP signaling is antagonized when phenotype switching is induced by plateletderived growth factor (PDGF) [5]. PDGF signaling decreases Trb3 expression and consequently leads to the down-regulation of Smad signal transducers. Trb3 is a BMPRIIinteracting protein and is released from BMPRII upon BMP stimulation, interacts with Smurf1, and leads to the degradation of Smurf1 through the ubiquitin-proteasome pathway [6]. The decrease in Smurf1 stabilizes molecules of the BMP pathway, such as Smad proteins, and potentiates the BMP response. The downregulation of Trb3 by PDGF leads to the reduced expression of Smad proteins and decreases in BMP and TGF $\beta$ signaling, thereby promoting the synthetic phenotype in VSMCs.

MicroRNAs (miRNAs) have been proposed as important mediators for the modulation of VSMC phenotype by repressing the targets implicated in regulation of VSMC phenotype [7]. Multiple miRNAs have been found to be responsible for VSMC differentiation and proliferation under physiological or pathological conditions [8]. Moreover, the expression of various miRNAs is modulated in response to extracellular signals, such as TGF $\beta$, BMP, PDGF, retinoic acid, and insulin [9]. For example, on BMP-induced signaling, Smad proteins translocate to the nucleus and modulate miRNA expression transcriptionally by directly associating with the promoter region of miR-302-367 gene cluster [10]. TGF $\beta$ and BMP also regulate the posttranscriptional processing of miRNAs, such as miR-21, through the association of Smads, primary miRNA (pri-miRNA), and a Drosha Microprocessor [11, 12]. miRNA-96 is an oncogenic miRNA as evidenced by the up-regulation of miR-96 in various types of cancer, such as bladder cancer, lung cancer and hepatocellular carcinoma $[13,14,15,16]$. This observation suggests that miR-96 may play a role in tumorigenesis or cell proliferation. Furthermore, miR-96 represses neural induction from human embryonic stem cells, suggesting that miR-96 may play a role in the maintenance of pluripotency or the self-renewal of embryonic stem cells [17]. In this study, we demonstrate that BMP signaling down-regulates transcription of miR-96, which in turn leads to the up-regulation of Trb3. We show that Trb3 is a novel target of miR-96. BMP-miR-96-mediated up-regulation of Trb3 in VSMCs results in increased contractile gene expression.

\section{Materials and Methods}

\section{Cell culture}

Human primary pulmonary artery smooth muscle cells (PASMCs) were purchased from Lonza (CC- 2581) and were maintained in Sm-GM2 medium (Lonza) containing 5\% fetal bovine serum (FBS). Recombinant human BMP4 and PDGF-BB were purchased from R\&D Systems. The cells were treated with $3 \mathrm{nM}$ BMP4 or $20 \mathrm{ng} / \mathrm{ml}$ PDGF-BB under starvation conditions $(0.2 \% \mathrm{FBS})$, as described previously [3]. 


\section{Quantitative reverse transcriptase-PCR (qRT-PCR)}

Quantitative analysis of the change in expression levels was calculated by a real-time PCR machine (iQ5; Bio-Rad) as described previously [5]. mRNA level was normalized to GAPDH. The real-time PCR primer sequences for pri-miR-96 were 5'CTGCCTTGAGTGCTCCTA-3' and 5'- CCTGACACAAGGATGCAG-3'. Primer sets for ASMA, CNN, SM22a, Id3, Smad4, and Trb3 have been published previously [5]. For detection of mature miRNA-96, a TaqMan MicroRNA assay kit (Applied Biosystems) was used according to the manufacturer's instructions. Data analysis was performed using a comparative $\mathrm{C}_{\mathrm{T}}$ method in Bio-Rad software. miRNA levels were normalized to U6 small nuclear RNA. The average of three experiments, each performed in triplicate, is presented with standard errors.

\section{miRNA mimic and anti-miRNA oligonucleotides}

Chemically modified double-stranded RNAs designed to mimic the endogenous mature miR-96 and negative control miRNA were purchased from Ambion. $2^{\prime}$ - $O$-methyl-modified RNA oligonucleotides complementary to miR-96 or green fluorescent protein (GFP; control) sequence were purchased from IDT. The sequences were: anti-miR-96, 5' AGCAAAAATGTGCTAGTGCCAAA 3'; anti-GFP, 5' AAGGCAAGCUGACCCUGAAGU-3' ${ }^{\prime}$. miRNA mimics and anti-miRNA oligonucleotides were transfected using RNAi Max (Invitrogen) according to the manufacturer's protocol at 5 $\mathrm{nM}$ and $40 \mathrm{nM}$, respectively.

\section{RNA interference}

Synthetic siRNA targeting human Smad4 (5'-

CCUGAGUAUUGGUGUUCCAUUGCUU-3') was purchased from Invitrogen. Nontargeting scrambled siRNA (\#1027280, Qiagen) was used as a control. The siRNAs were transfected at $40 \mathrm{nM}$ using RNAi Max (Invitrogen), according to the manufacturer's protocol.

\section{Luciferase reporter constructs}

The full-length 3'UTR sequence of Trb3 and the predicted MRE sequence were cloned into the pIS0 vector (Addgene) containing the luciferase gene. The full-length 3'UTR sequence of Trb3 was amplified by RT-PCR from mRNAs isolated from PASMCs using 5'-

ATGGAGCTCTAGGACCACCCTACTACACG-3' and 5'ATCGGCCGGCCCCTTTATTAGGCACAGGTAAA-3'. 5'AAGGGAGGTATCCCTGTGCCAAA-3' and 5' - TTTGGCACAGGGATACCTCCCTT-3' were used for MRE and 5'- AAGGGAGGTATCCCTGTCGGTAA-3' and 5'TTACCGACAGGGATACCTCCCTT-3' were used for the MRE mutant (MRE-mut).

\section{Luciferase Assay}

Cos7 cells were transfected with luciferase reporter constructs using Fugene HD (Roche) and a ßgalactosidase (ß-gal) expression plasmid as an internal transfection control. Twentyfour hours later, cells were transfected with $5 \mathrm{nM}$ miR-96 mimic or control miRNA mimic 
using RNAi Max (Invitrogen). Luciferase assays were carried out and luciferase activities were presented after normalization to ßgalactosidase activities.

\section{Immunoblotting}

Cells were lysed in TNE buffer and total cell lysates were separated by SDS-PAGE, transferred to PVDF membranes (Millipore), immunoblotted with antibodies, and visualized using an enhanced chemiluminescence detection system (Amersham Biosciences). Antibodies used for immunoblotting were an anti-GAPDH antibody (2E3-2E10, Abnova) and an anti-human Trb3 antibody (AP8157a, Abgent).

\section{Statistical Analysis}

The results presented were an average of at least three experiments that were each performed in triplicate with standard errors. Statistical analyses were performed by an analysis of variance followed by Student's $t$ test, using Prism 4 software (GraphPAD Software Inc.). $P$ values of $<0.05$ were considered significant and are indicated with asterisks.

\section{Results}

\section{BMP4 down-regulates the expression of miR-96}

A miRNA expression profiling analysis in pulmonary artery smooth muscle cells (PASMCs) indicated that miR-96 is repressed upon BMP4 stimulation (3 nM) for $24 \mathrm{hr}$ (data not shown). qRT-PCR analysis of PASMCs confirmed the miRNA expression profiling result and showed a decrease in miR- 96 to 50\% of the basal level upon BMP4 stimulation for 24 $\mathrm{hr}$ (Figure 1A). To determine whether transcription of miR-96 is affected by BMP4, the primiR-96 expression level was examined. A timecourse analysis of pri-miR-96 showed a rapid reduction of pri-miR-96 within $2 \mathrm{hr}$ after BMP4 stimulation, indicating that the downregulation of miR-96 may be controlled transcriptionally (Figure 1B). To examine whether the repression of pri-miR-96 by BMP4 was mediated by the signal transducer of the BMP signaling pathway, Smad4 was down-regulated by small interfering RNA (siRNA) and the levels of pri-miR-96 were examined (Figure 1C). The repression of pri-miR-96 by BMP4 was inhibited when Smad4 was down-regulated, suggesting that down-regulation of miR-96 by BMP4 is dependent on the signal transducer of the BMP signaling pathway, Smad4.

\section{miR-96 regulates SMC-specific gene expression}

We investigated a role for miR-96 in VSMCs. VSMCs were transfected with anti-miR-96 and the levels of SMC-specific genes, such as ASMA, CNN and SM22a, and a transcription target of BMP signaling, Id3, were examined (Figure 2A). The expression of SMC-specific genes and Id3 was enhanced in anti-miR-96-transfected cells in comparison with control cells, suggesting that endogenous miR-96 inhibits the induction of the contractile phenotype of VSMC characterized by an increased expression of the SMC-specific genes. Furthermore, the induction of SMC-specific genes upon BMP4 stimulation was significantly blocked in miR-96 mimic-transfected cells in comparison with control cells; however, miR-96 itself does not seem to be sufficient to induce the expression of contractile genes (Figure 2B). These results demonstrate that the down-regulation of miR-96 is essential for the induction of SMC-specific genes, leading to the contractile phenotype of VSMCs. 


\section{Trb3 is a novel target of miR-96}

In a search for potential targets of miR-96 using the TargetScan target prediction algorithm, we observed an evolutionarily conserved miRNA recognition element (MRE) partially complementary to miR-96 in the 3' UTR of tribbles homolog 3 (Trb3) gene (Figure 3A). Trb3 is a critical molecule for regulatory mechanism of the BMP signaling pathway that functions as a positive regulator. To assess whether endogenous miR-96 regulates the expression of Trb3, miR-96 was inhibited by the transfection of antisense oligonucleotides complementary to the miR-96 (anti-miR-96) in PASMCs, and the relative expression level of $T r b 3$ to $G A P D H$ was examined (Figure 3B). The antisense oligonucleotides complementary to $G F P$ (anti-GFP) were used as controls. $\operatorname{Tr} b 3$ expression increases approximately 3.5 -fold when endogenous miR-96 is inhibited, indicating that endogenous miR-96 represses $\operatorname{Tr} b 3$ expression. Conversely, exogenous miR-96 down-regulates endogenous Trb3 expression levels (Figure 3C). In VSMCs transfected with $5 \mathrm{nM}$ miR-96 mimic, the relative mRNA level of $\operatorname{Tr} b 3$ to $G A P D H$ is reduced to approximately $40 \%$ compared with the $5 \mathrm{nM}$ control miRNA-transfected VSMCs. Moreover, immunoblot analyses indicated that the endogenous Trb3 protein in PASMCs was down-regulated upon miR-96 over-expression in comparison with control miRNA mimic (Figure 3D). These results indicate that Trb3 is a novel target of miR-96.

To confirm that miR-96 targets Trb3, we measured the expression of the luciferase reporter construct containing the 3'UTR of $\operatorname{Tr} b 3$ at the downstream of the luciferase reporter gene in the presence of miR-96 mimic (Figure 3E). Consistent with the qRT-PCR results, the luciferase activity of the Trb33'UTR construct was significantly reduced upon expression of the miR-96 mimic, demonstrating that Trb3 is a target of miR-96. To determine whether miR-96 targets Trb3 through the evolutionarily conserved MRE in the 3' UTR of Trb3 gene, luciferase reporter containing MRE was generated. miR- 96 reduced the luciferase activity of MRE by approximately 50\%. Mutations in MRE, which disrupted base pairing with miR-96 (Figure 3F), abrogated the inhibition of luciferase activity by miR-96, suggesting that the conserved MRE is a critical target site for recognition of Trb3 mRNA by miR-96.

\section{miR-96 is not affected by PDGF signaling}

Trb3 is known to be an essential positive regulator of the BMP-mediated signaling pathway to modulate VSMC phenotype [6]. Moreover, it has been shown that Trb3 is down-regulated by miR-24 upon PDGF signaling which promotes the synthetic phenotype of VSMCs [5]. We confirmed that the $\operatorname{Tr} 33$ mRNA level is down-regulated to approximately $40 \%$ of the basal level of the control after $24 \mathrm{hr}$ of PDGF-BB stimulation (Figure 4A). To test the possibility that PDGF-BB induces miR-96 to downregulate Trb3, mature miR-96 levels in VSMCs were examined by qRT-PCR following PDGF-BB stimulation. PDGF signaling had no effect on the expression of miR-96, suggesting that miR-96 is modulated only by BMP signaling, not PDGF-BB (Figure 4B).

\section{Discussion}

In this study, we elucidated a novel mechanism by which BMP signaling promotes the contractile phenotype of VSMCs. BMP4-dependent down-regulation of miR-96 elevates the 
expression of Trb3, which in turn leads to potentiate the BMP signaling pathway (Figure 4C). We identified Trb3 as a novel target of miR-96 and provided evidence that downregulation of miR-96 is critical for promoting the contractile VSMC phenotype by inducing the SMC-specific contractile gene expression. The aberrant regulation of the VSMC phenotype, in particular a switch from the contractile to the synthetic phenotype, is a critical phenomenon underlying the pathogenesis of a variety of vascular proliferative diseases, including PAH. Differential expression of miRNAs responsible for the regulation of the VSMC phenotype has been demonstrated in the pulmonary vasculature of patients with PAH or other vascular proliferative conditions [18]. Therefore, the modulation of the miRNA level in vivo by delivery of anti-miR oligonucleotides or adeno-associated virus is considered a novel therapy. For example, miR-145 and miR-21 are dysregulated in the mouse model of PAH $[19,20]$. Down-regulation of miR-145 prevents the development of $\mathrm{PAH}$, while down-regulation of miR-21 promotes the development of PAH. In addition, miR-424 and miR-503 are down-regulated in PAH and restoring the expression of miR-424 ameliorates PAH in experimental models [21].

We demonstrate that miR-96 level is modulated by BMP4 and affects the regulation of the VSMC phenotype. miR-96 inhibits the induction of contractile phenotype by BMP signaling. However, further investigation remains to determine whether miR-96 expression is altered in cardiovascular diseases. Differential expression and regulation of miR-96 have been observed during the differentiation of various cell types. For example, miR-96 is differentially expressed during osteogenesis, adipogenesis, and neurogenesis [17, 22, 23]. miR-96 expression was increased during osteogenic and adipogenic induction of human bone marrow-derived MSCs [23]. However, miR-96 was uniquely down-regulated in neuroectodermal precursor cells and induced neuronal differentiation through regulating its target, PAX6, a key transcription factor of neural induction [17]. Furthermore, an increased expression of miR-96 has been observed in various tumors such as bladder cancer and colorectal cancer, suggesting that miR-96 may be involved in a relatively common mechanism during tumorigenesis $[24,25]$. Therefore, miR-96 may be critical for a primary regulatory mechanism governing basic cellular functions, cell differentiation and tumorigenesis.

We identified Trb3 as a novel target of miR-96. Trb3 has been shown to play a critical role in the regulation of various signaling pathways. For example, Trb3 inhibits Akt activity and impairs insulin signaling in hepatic cells and in $\mathrm{C} 2 \mathrm{C} 12$ muscle cells $[8,26]$. Trb3 also mediates ER stress-induced insulin resistance in skeletal muscle through regulation of programmed cell death [27]. In the BMP signaling pathway, Trb3 is known to mediate the degradation of Smurf1, which facilitates the degradation of Smads and RhoA. Smads and RhoA are downstream signal transducers critical for mediating the pro-contractile signal from the BMP signaling pathway in VSMCs [6]. Thus, we speculate that the induction of Trb3 by down-regulation of miR-96 leads to pro-contractile signaling via multiple mechanisms through the reduction of Smurf1.

In previous studies, $\operatorname{Trb} 3$ has been shown to mediate the BMP-dependent regulation of SMC-specific gene expression [6]. The PDGF signaling pathway induces miR-24, which targets Trb3 [5]. Repression of Trb3 results in a decrease in BMP signaling, promoting the 
synthetic phenotype in VSMCs. Therefore, we investigated a potential crosstalk between miR-96 and PDGF signaling by monitoring the effect of PDGF-BB on the level of miR-96. We did not observe significant changes in the expression of miR-96 upon PDGF-BB stimulation, suggesting that miR-96 is not regulated by PDGF at the level of transcription or processing in VSMCs. Therefore, the regulatory mechanism of miR-96 by BMP is specific and miR-96 does not play a role in PDGF-mediated regulation of the synthetic phenotype.

\section{Acknowledgments}

This work was supported by the Incheon National University International Research Grant in 2012 and the Basic Science Research Program through the National Research Foundation of Korea (NRF) funded by the Ministry of Education, Science and Technology (2012R1A1A1042812) to HK.

\section{References}

1. Owens GK, Kumar MS, Wamhoff BR. Molecular regulation of vascular smooth muscle cell differentiation in development and disease. Physiol Rev. 2004; 84:767-801. [PubMed: 15269336]

2. Monzen K, Nagai R, Komuro I. A role for bone morphogenetic protein signaling in cardiomyocyte differentiation. Trends Cardiovasc Med. 2002; 12:263-269. [PubMed: 12242050]

3. Lagna G, Ku MM, Nguyen PH, Neuman NA, Davis BN, Hata A. Control of phenotypic plasticity of smooth muscle cells by bone morphogenetic protein signaling through the myocardin-related transcription factors. J Biol Chem. 2007; 282:37244-37255. [PubMed: 17947237]

4. ten Dijke P, Arthur HM. Extracellular control of TGFbeta signalling in vascular development and disease. Nat Rev Mol Cell Biol. 2007; 8:857-869. [PubMed: 17895899]

5. Chan MC, Hilyard AC, Wu C, Davis BN, Hill NS, Lal A, Lieberman J, Lagna G, Hata A. Molecular basis for antagonism between PDGF and the TGFbeta family of signalling pathways by control of miR-24 expression. EMBO J. 2010; 29:559-573. [PubMed: 20019669]

6. Chan MC, Nguyen PH, Davis BN, Ohoka N, Hayashi H, Du K, Lagna G, Hata A. A novel regulatory mechanism of the bone morphogenetic protein (BMP) signaling pathway involving the carboxyl-terminal tail domain of BMP type II receptor. Mol Cell Biol. 2007; 27:5776-5789. [PubMed: 17576816]

7. Hata A. Functions of MicroRNAs in Cardiovascular Biology and Disease. Annu Rev Physiol.

8. Kato S, Du K. TRB3 modulates C2C12 differentiation by interfering with Akt activation. Biochem Biophys Res Commun. 2007; 353:933-938. [PubMed: 17207467]

9. Kang H, Hata A. MicroRNA regulation of smooth muscle gene expression and phenotype. Curr Opin Hematol. 19:224-231.

10. Kang H, Louie J, Weisman A, Sheu-Gruttadauria J, Davis-Dusenbery BN, Lagna G, Hata A. Inhibition of MicroRNA-302 (miR-302) by Bone Morphogenetic Protein 4 (BMP4) Facilitates the BMP Signaling Pathway. J Biol Chem. 287:38656-38664.

11. Davis BN, Hilyard AC, Lagna G, Hata A. SMAD proteins control DROSHA-mediated microRNA maturation. Nature. 2008; 454:56-61. [PubMed: 18548003]

12. Kang H, Davis-Dusenbery BN, Nguyen PH, Lal A, Lieberman J, Van Aelst L, Lagna G, Hata A. Bone morphogenetic protein 4 promotes vascular smooth muscle contractility by activating microRNA-21 (miR-21), which down-regulates expression of family of dedicator of cytokinesis (DOCK) proteins. J Biol Chem. 287:3976-3986.

13. Han Y, Chen J, Zhao X, Liang C, Wang Y, Sun L, Jiang Z, Zhang Z, Yang R, Chen J, Li Z, Tang A, Li X, Ye J, Guan Z, Gui Y, Cai Z. MicroRNA expression signatures of bladder cancer revealed by deep sequencing. PLoS One. 2011; 6:e18286. [PubMed: 21464941]

14. Ma L, Huang Y, Zhu W, Zhou S, Zhou J, Zeng F, Liu X, Zhang Y, Yu J. An integrated analysis of miRNA and mRNA expressions in non-small cell lung cancers. PLoS One. 2011; 6:e26502. [PubMed: 22046296]

15. Chen RX, Xia YH, Xue TC, Ye SL. Suppression of microRNA-96 expression inhibits the invasion of hepatocellular carcinoma cells. Mol Med Rep. 2012; 5:800-804. [PubMed: 22160187] 
16. Navon R, Wang H, Steinfeld I, Tsalenko A, Ben-Dor A, Yakhini Z. Novel rank-based statistical methods reveal microRNAs with differential expression in multiple cancer types. PLoS One. 2009; 4:e8003. [PubMed: 19946373]

17. Du ZW, Ma LX, Phillips C, Zhang SC. miR-200 and miR-96 families repress neural induction from human embryonic stem cells. Development. 2013; 140:2611-2618. [PubMed: 23637338]

18. McDonald RA, Hata A, MacLean MR, Morrell NW, Baker AH. MicroRNA and vascular remodelling in acute vascular injury and pulmonary vascular remodelling. Cardiovasc Res. 2012; 93:594-604. [PubMed: 22065733]

19. Caruso P, Dempsie Y, Stevens HC, McDonald RA, Long L, Lu R, White K, Mair KM, McClure JD, Southwood M, Upton P, Xin M, van Rooij E, Olson EN, Morrell NW, MacLean MR, Baker AH. A role for miR-145 in pulmonary arterial hypertension: evidence from mouse models and patient samples. Circ Res. 2012; 111:290-300. [PubMed: 22715469]

20. Caruso P, MacLean MR, Khanin R, McClure J, Soon E, Southgate M, MacDonald RA, Greig JA, Robertson KE, Masson R, Denby L, Dempsie Y, Long L, Morrell NW, Baker AH. Dynamic changes in lung microRNA profiles during the development of pulmonary hypertension due to chronic hypoxia and monocrotaline. Arterioscler Thromb Vasc Biol. 2010; 30:716-723. [PubMed: 20110569]

21. Kim J, Kang Y, Kojima Y, Lighthouse JK, Hu X, Aldred MA, McLean DL, Park H, Comhair SA, Greif DM, Erzurum SC, Chun HJ. An endothelial apelin-FGF link mediated by miR-424 and miR-503 is disrupted in pulmonary arterial hypertension. Nat Med. 2013; 19:74-82. [PubMed: 23263626]

22. Suomi S, Taipaleenmaki H, Seppanen A, Ripatti T, Vaananen K, Hentunen T, Saamanen AM, Laitala-Leinonen T. MicroRNAs regulate osteogenesis and chondrogenesis of mouse bone marrow stromal cells. Gene Regul Syst Bio. 2008; 2:177-191.

23. Laine SK, Alm JJ, Virtanen SP, Aro HT, Laitala-Leinonen TK. MicroRNAs miR-96, miR-124, and miR-199a regulate gene expression in human bone marrow-derived mesenchymal stem cells. J Cell Biochem. 2012; 113:2687-2695. [PubMed: 22441842]

24. Yoshino H, Seki N, Itesako T, Chiyomaru T, Nakagawa M, Enokida H. Aberrant expression of microRNAs in bladder cancer. Nat Rev Urol. 2013; 10:396-404. [PubMed: 23712207]

25. Xu XM, Qian JC, Deng ZL, Cai Z, Tang T, Wang P, Zhang KH, Cai JP. Expression of miR-21, miR-31, miR-96 and miR-135b is correlated with the clinical parameters of colorectal cancer. Oncol Lett. 2012; 4:339-345. [PubMed: 22844381]

26. Du K, Herzig S, Kulkarni RN, Montminy M. TRB3: a tribbles homolog that inhibits Akt/PKB activation by insulin in liver. Science. 2003; 300:1574-1577. [PubMed: 12791994]

27. Ohoka N, Yoshii S, Hattori T, Onozaki K, Hayashi H. TRB3, a novel ER stress-inducible gene, is induced via ATF4-CHOP pathway and is involved in cell death. EMBO J. 2005; 24:1243-1255. [PubMed: 15775988] 
A

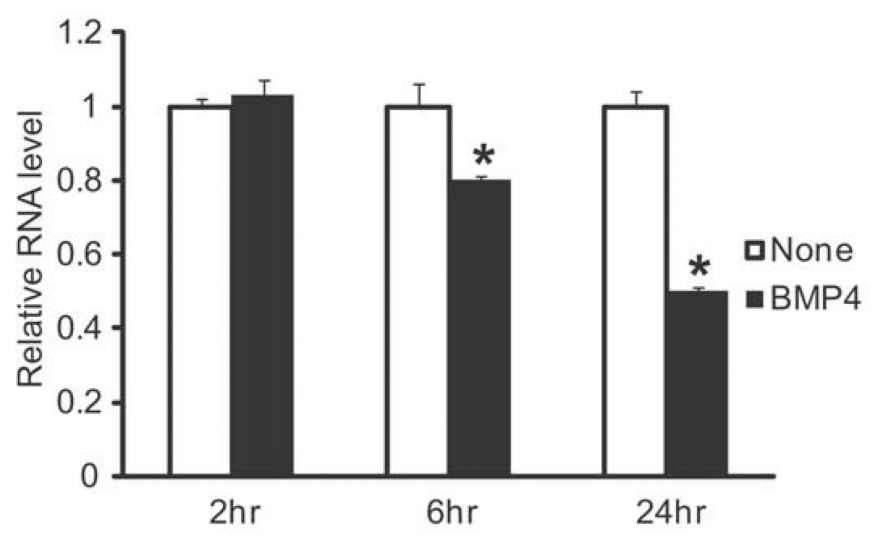

C

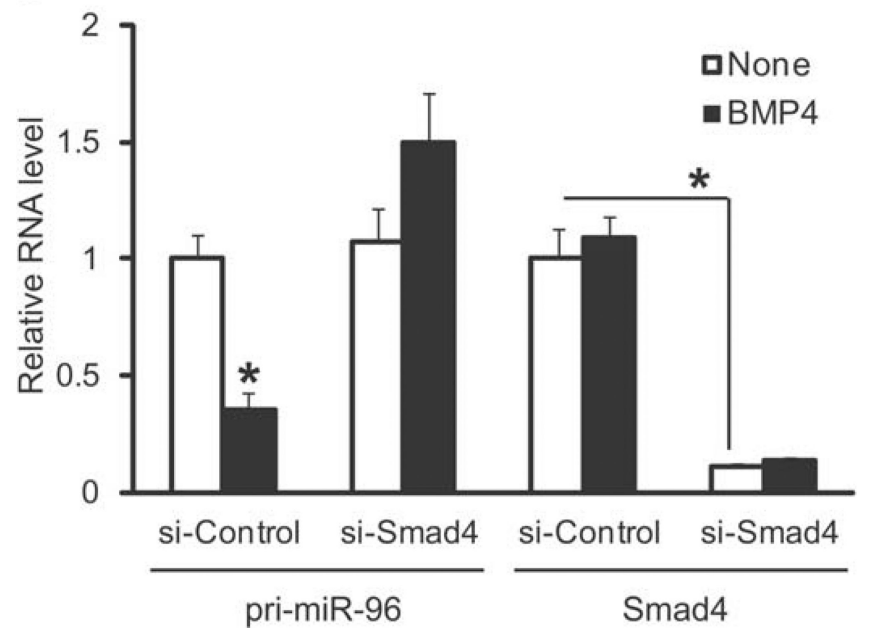

B

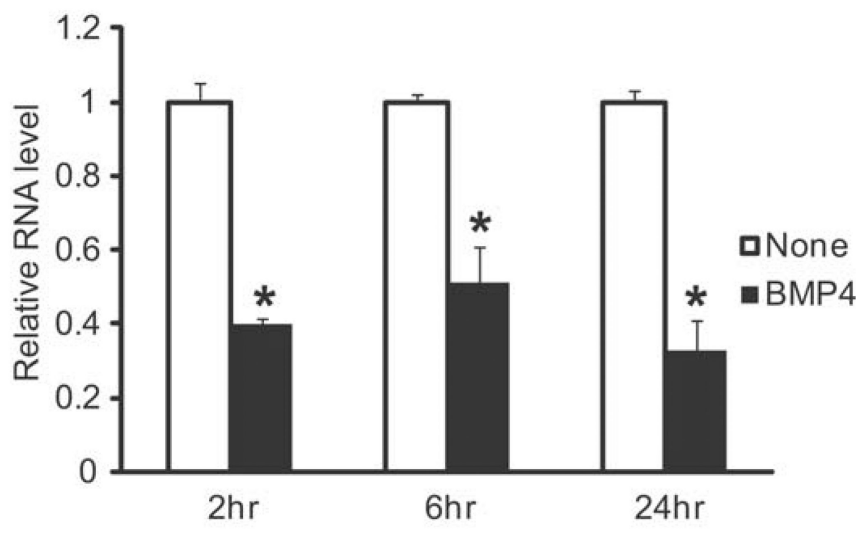

Figure 1. miR-96 is regulated by BMP4 signaling

A. Expression levels of miR-96 normalized to U6 snRNA were examined by qRT-PCR in PASMCs stimulated with $3 \mathrm{nM}$ BMP4 for a period of time as indicated. Data represent the means \pm S.E. of triplicates. *, $p<0.001$. B. Expression levels of primiR- 96 normalized to GAPDH were examined by qRT-PCR in PASMCs stimulated with $3 \mathrm{nM}$ BMP4 for a period of time as indicated. Data represent the means \pm S.E. of triplicates. $*$, p $<0.01$. C. PASMCs were transfected with non-targeting control siRNA (si-Control) and siRNA against Smad4 (si-Smad4), followed by $3 \mathrm{nM}$ BMP stimulation for $24 \mathrm{hr}$. Levels of pri-miR-96 and Smad4 mRNA relative to GAPDH were measured by qRT-PCR. Data represent the means \pm S.E. of triplicates. *, p $<0.001$. 
A

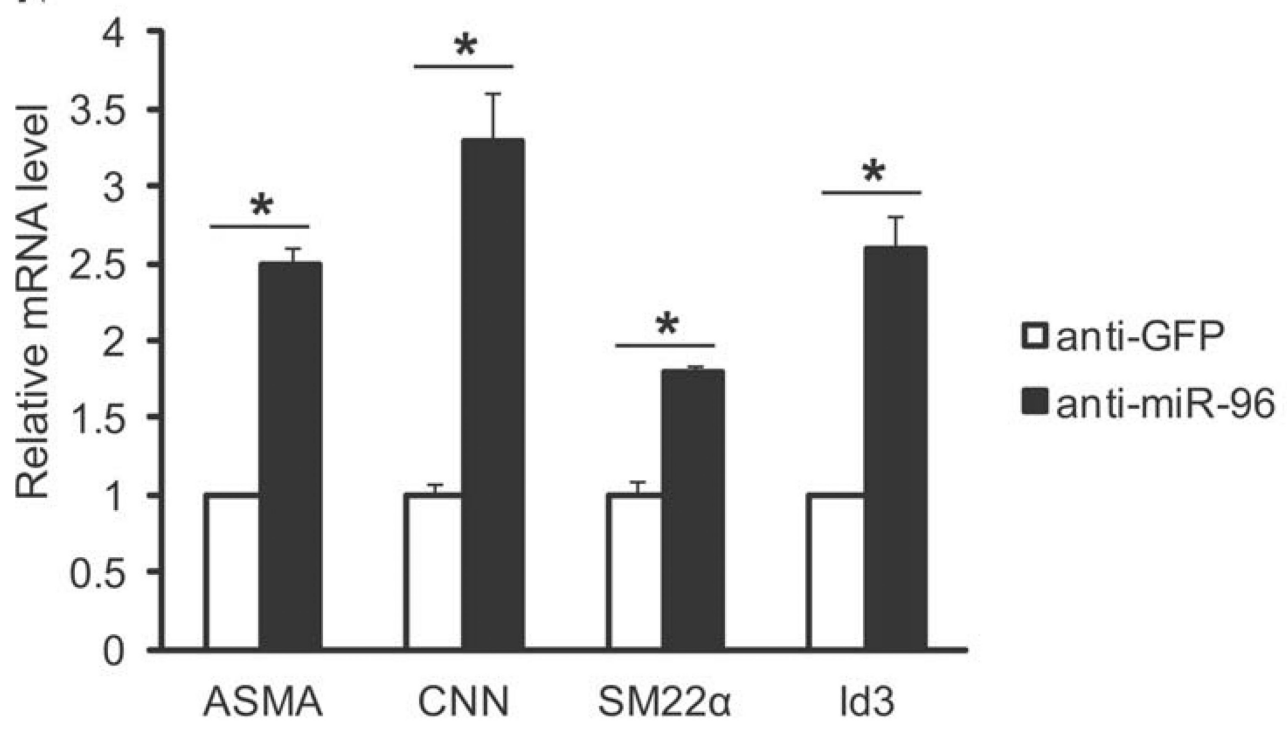

B

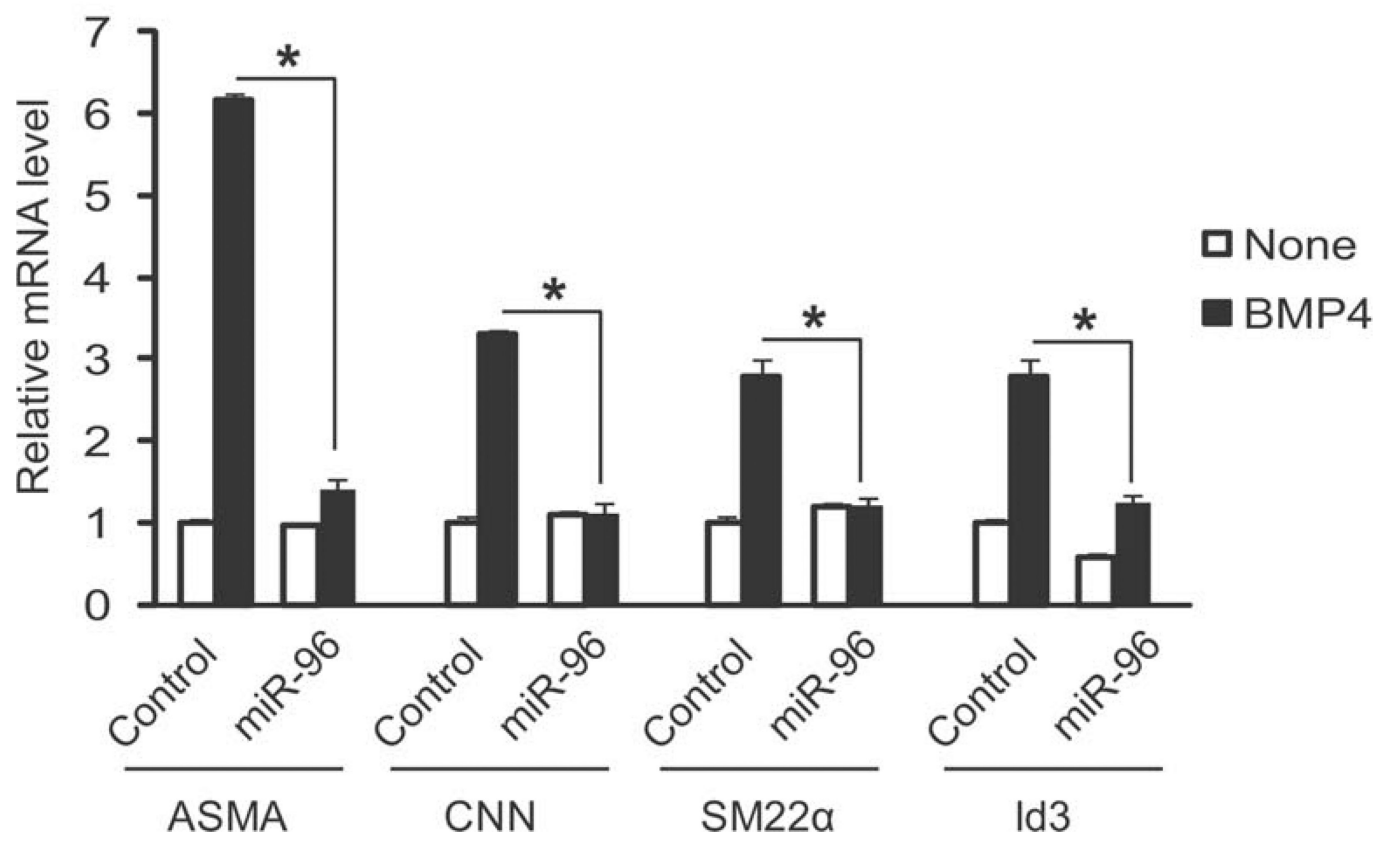

Figure 2. miR-96 is critical for the BMP4-dependent induction of SMC-specific genes A. The expression levels of SMC-specific genes (ASMA, CNN, SM22a) and Id3 normalized to GAPDH were examined by qRT-PCR in PASMCs transfected with $50 \mathrm{nM}$ antisense oligonucleotides to GFP (anti-GFP) or miR-96 (anti-miR-96). Data represent the means \pm S.E. of triplicates. *, $p<0.01$. B. PASMCs were transfected with negative control mimic or miR-96 mimic $(5 \mathrm{nM})$. Cells were then treated with BMP4 for $24 \mathrm{hr}$ and subjected to qRT-PCR analysis of SMC-specific genes and Id3. Data represent the means \pm S.E. of triplicates. *, $\mathrm{p}<0.001$. 
A

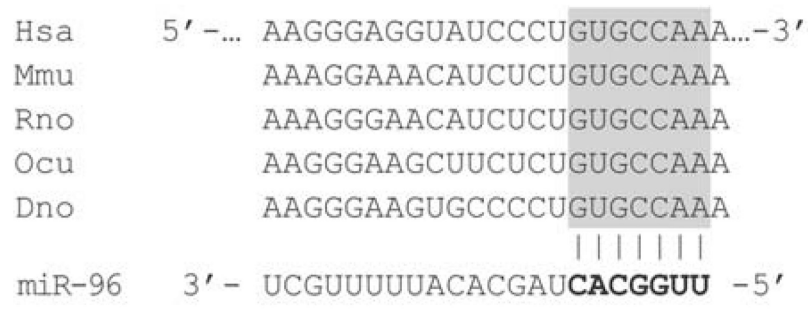

B

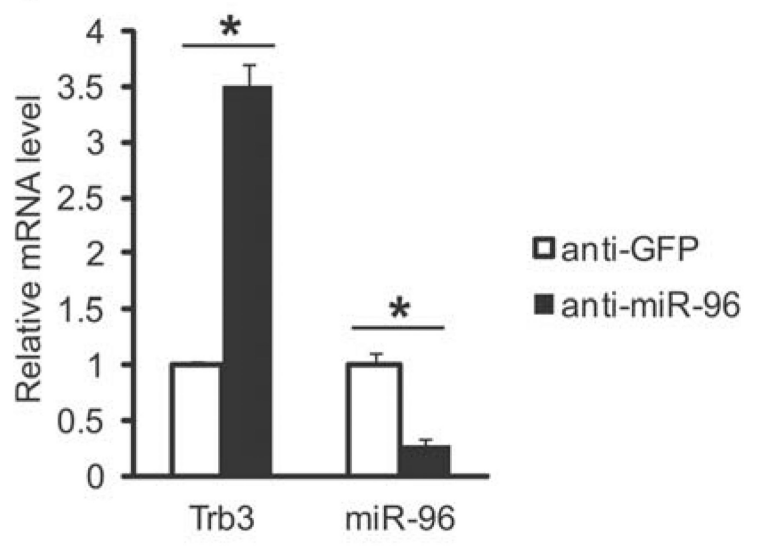

E

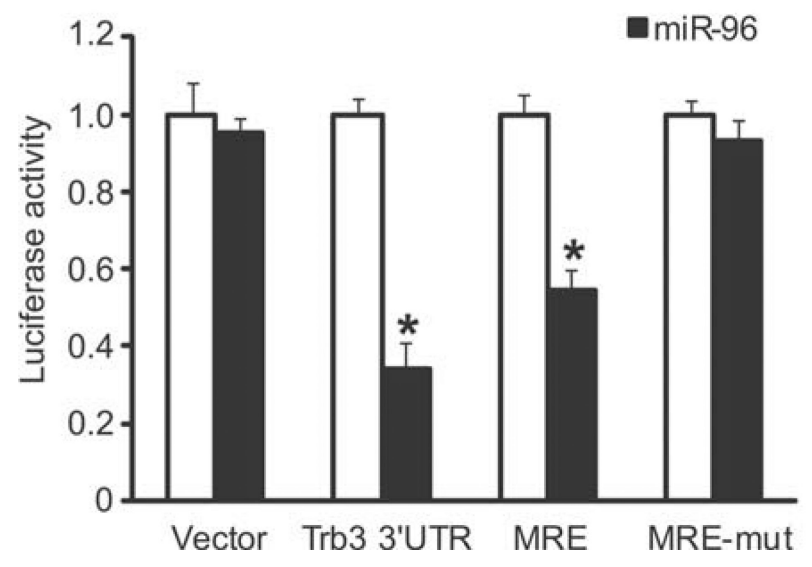

C
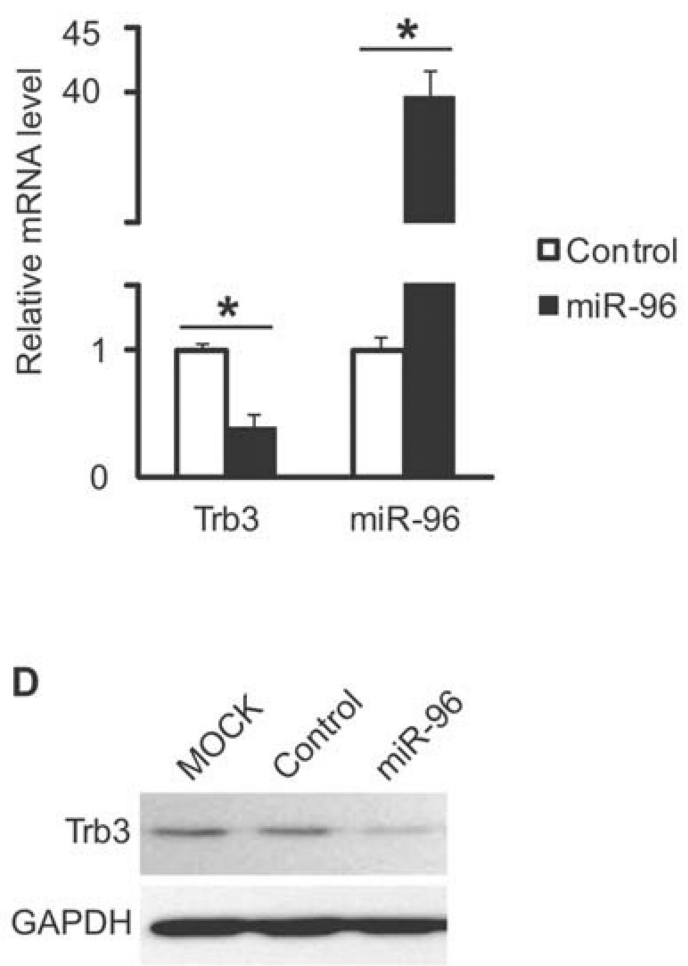

$\mathbf{F}$

MRE 5' AAgGGAGGUAUCCCUGUGCCAAA 3' 1111111 miR-96 3' UCGUUUUUACACGAUCACGGUUU 5' II I MRE-mut 5' AAGGGAGGUAUCCCUGUCGGTAA 3'

Figure 3. miR-96 targets Trb3

A. miR-96 MRE sequence found in the 3' UTR of Trb3 mRNA is evolutionarily conserved. Has: human, Mmu: mouse, Rno: rat, Ocu: rabbit, Dno: armadillo. B. Levels of Trb3 mRNA relative to GAPDH and miR-96 relative to U6 snRNA were quantified by qRT-PCR analysis in PASMCs transfected with $50 \mathrm{nM}$ anti-GFP or anti-miR-96. Data represent the means \pm S.E. of triplicates. *, p<0.001. C. Levels of Trb3 mRNA relative to GAPDH and miR-96 relative to U6 snRNA were examined by qRT-PCR analysis in PASMCs transfected with 5 $\mathrm{nM}$ negative control mimic or miR-96 mimic. Data represent the means \pm S.E. of triplicates. 
*, $\mathrm{p}<0.001$. D. Total cell lysates of PASMCs transfected with $5 \mathrm{nM}$ control mimic or miR-96 mimic for $48 \mathrm{hr}$ were subjected to immunoblot analysis of endogenous Trb3 and GAPDH. E. Luciferase activities of constructs with full-length 3'UTR of Trb3, wild type Trb3 MRE (MRE) or mutated Trb3 MRE (MRE-mut) were examined in Cos7 cells by transfecting control or miR-96 mimic. Data represent the means \pm S.E. of triplicates. ${ }^{*}, \mathrm{p}<0.01$. F. Sequence of wild type miR-96 MRE and mutated MRE (MRE-mut) cloned into the 3'UTR of the luciferase gene is shown. 
C

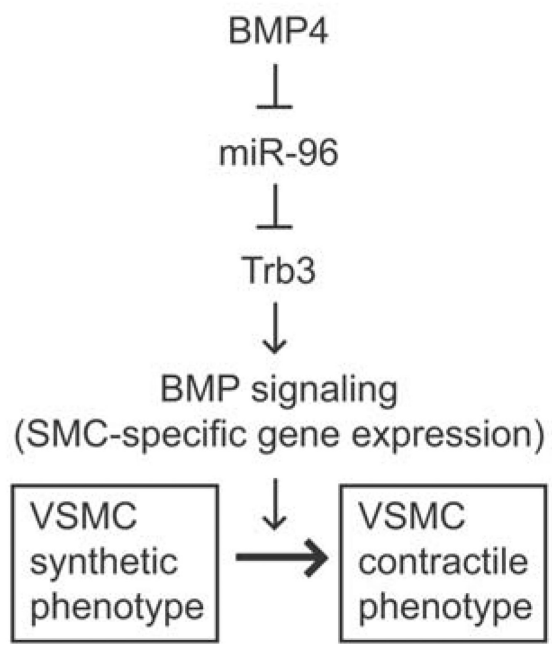

A

B
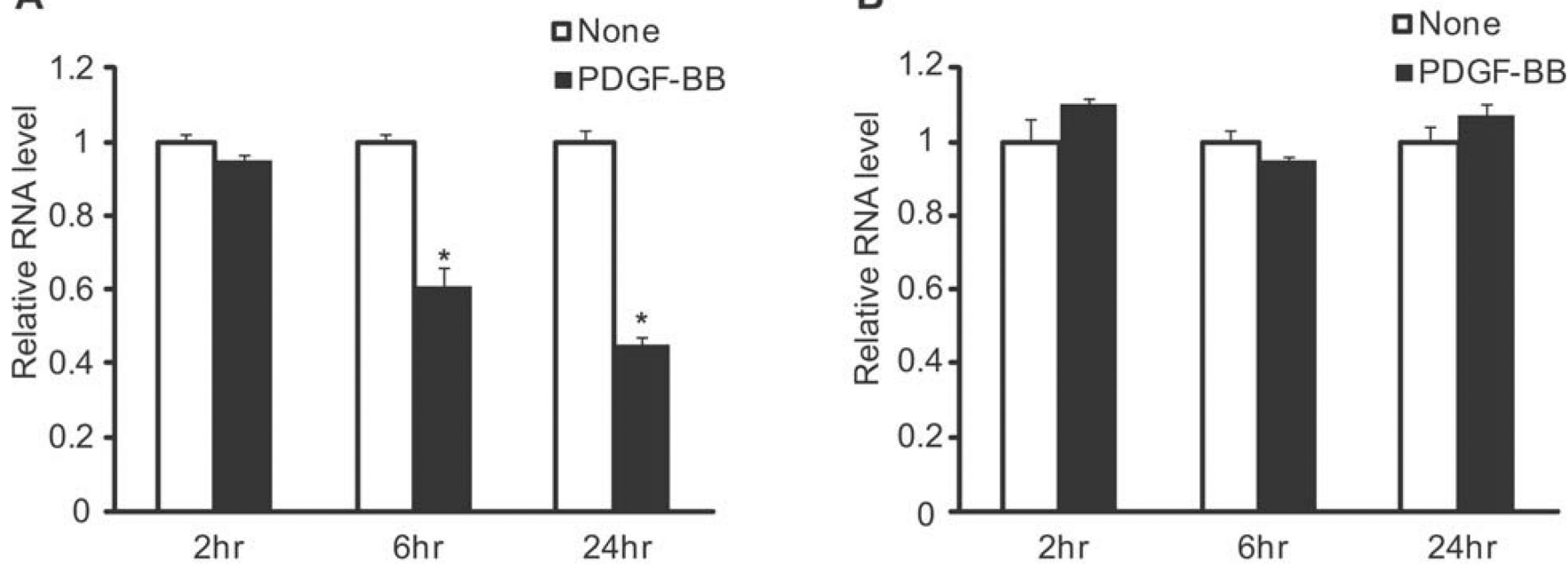

Figure 4. miR-96 is not affected by PDGF signaling

A. Expression levels of Trb3 mRNA normalized to GAPDH were examined by qRT-PCR in PASMCs stimulated with $20 \mathrm{ng} / \mathrm{ml}$ PDGF-BB for time as indicated. Data represent the means \pm S.E. of triplicates. * ${ }^{*}<<0.01$. B. Expression levels of miR-96 normalized to U6 snRNA were examined by qRT-PCR in PASMCs stimulated with $20 \mathrm{ng} / \mathrm{ml}$ PDGF-BB for time as indicated. Data represent the means \pm S.E. of triplicates. C. Schematic diagram of BMP signaling pathway mediated by down-regulation of miR-96 in VSMCs. 